\title{
Independence and Uniqueness of the Mixed-Strategy Equilibrium in Social Networks
}

\section{Darong Dai ${ }^{1}$}

\begin{abstract}
We develop topological analysis of social-network effect on game equilibrium in the context of two-player asymmetric normal-form games and also in evolutionary sense. Firstly, it is confirmed that the game equilibrium in many social networks cannot be established through that in a well-mixed population. In other words, we have proved the independence of the mixedstrategy equilibrium in social networks. Secondly, it is demonstrated that the game equilibrium exhibits injective property with respect to the corresponding social-network effect under consideration. That is, the uniqueness of the mixed-strategy game equilibrium in a given social network is identified. Thirdly, it is argued that uniqueness implies independence for a wide range of social networks and we have even derived the biggest sets of social networks in which independence and uniqueness hold true, respectively, in the underlying game. To sum up, we have provided qualitative characterizations about topological properties of the mixed-strategy game equilibrium in general social networks.
\end{abstract}

Keywords: social network, asymmetric game, mixed-strategy equilibrium, independence, uniqueness

\section{JEL Classification: C62, C72}

\section{Introduction}

Noting that social networks have been paid heavy attention to in recent studies, including economics (see, Bandiera and Rasul, 2006; Goyal, 2007; Acemoglu et al., 2010, 2011, 2012; Golub and Jackson, 2010), biology (e.g., Nowak, 2006; Ohtsuki et al., 2006; Pacheco et al., 2008; Tarnita et al., 2009; Fu et al., 2010; Allen et al., 2012), sociology (Zhang, 2004; Jackson, 2008) and physics (Pacheco et al., 2006; Ohtsuki et al., 2007), and the theory about game equilibrium in well-mixed populations has been well-established (see, Weibull, 1995; Hofbauer and Sigmund, 2003), the present paper is encouraged to discuss the question that whether or not we can use the game equilibrium derived in well-

1 Department of Economics, School of Business, Nanjing University, Nanjing 210093, People's Republic of China. daidarong998@163.com 
mixed populations to effectively approximate the equilibrium of non-trivial social networks. Accordingly, the major goal of the paper is to illustrate that social-network structure does affect the resulting game equilibrium, which is a mixed-strategy equilibrium in general, and we further provide the explicit dimensional-constraint under which the conclusion holds with certain stability and also in generic sense (e.g., Mas-Colell and Nachbar, 1991). However, it is worth noting that we just consider exogenous social-network effect rather than the endogenous formation of social networks discussed in Skyrms and Pemantle (2000), Bala and Goyal (2000) and Galeotti et al. (2006). To the best of our knowledge, the paper, for the first time, investigates the topology of mixed-strategy game equilibrium in general social networks.

Why do we focus on social-network effect? In the traditional approach of evolutionary game theory, individuals are usually assumed to meet at random and hence the well-known random-matching rule (e.g., Maynard Smith, 1982; Fudenberg and Levine, 1993; Ellison, 1994; Okuno-Fujiwara and Postlewaite, 1995; Weibull, 1995) is widely employed. In a wellmixed population, this methodology indeed provides us with an appropriate benchmark. Nonetheless, it is convincing to argue that people live in a highly structured society consists of groups, which implies that random matching will not always provide us with compelling approximation to reality when we are concerned with local interactions rather than uniform interactions among the players. In fact, Ellison (1993) shows that local interaction has very important and also different implications in equilibrium selection relative to that of uniform interaction or random matching. With the good purpose of correctly predicting the behavior of individuals, it is absolutely necessary to introduce social-network structure induced by non-uniform social interactions (see, Haag and Lagunoff, 2006; Horst and Scheinkman, 2006) into our games.

Indeed, many existing studies have been devoted to this issue. For example, noting that personal interactions among individuals are structured by families, neighborhoods, communities, and markets, as well as other formal and informal institutions, most of existing articles emphasize reputation effect and retaliation effect of the community (see, Kandori, 1992; Kahneman et al., 1986; Ghosh and Ray, 1996; Spagnolo, 1999; Anderson and Smith, 2010; Takahashi, 2010), while in the model of Bowles and Gintis (1998), the segmentation or segregation effect (e.g., Schelling, 1969, 1971) of the community is also explored. Undoubtedly, all of these effects can be regarded as specific examples of the general social-network effect discussed in the paper. Furthermore, individuals in the games also have preferences, motivations and emotions, that is, they have control over the frequency or duration of interactions. For instance, studies of dynamic social networks and theories on the evolution of cooperation in dynamically structured populations (e.g., Ohtsuki et al., 2007; Pacheco et al., 2008; Pacheco et al., 2006) usually construct models in which individuals differ in the rate at which they seek new interactions with others.

For the sake of simplicity, we have interpreted social-network effect in the sense of Skyrms and Pemantle (2000) that the frequencies individuals meet each other are modified by the existing social-network structure when compared to that of well-mixed populations. That is, in evolutionary sense, social network affects the game equilibrium through the 
impact forced on the frequencies individuals meet each other and hence the payoffs individuals finally receive. And it is easily seen that our specification is without loss of generality. In our model, representative players are assumed to maximize the discounted payoffs subject to the belief-learning dynamics, and then the solutions, if exist, form the game equilibrium.

The main result reveals that, in many interesting and also important cases, one can hardly approximate the game equilibrium in social network via the game equilibrium of an ideal random-matching world. In other words, it is reasonable to argue that the game equilibrium in nontrivial social networks would be of independent interest. Moreover, it is illustrated that the game equilibrium is indeed injective map with respect to the socialnetwork effect under consideration. What's the corresponding inspiration? Rather, we may interpret the result as that different social networks yield their independent interest if they produce different (in the sense of our specification in the model) social-network effects. In other words, the uniqueness of the mixed-strategy game equilibrium in a given social network is identified.

The paper is organized as follows. Section 2 presents the basic model. In section 3, we mainly analyze the social-network effect imposed on the game equilibrium defined and derived in section 2. And our major innovations appear in section 3. Section 4 concludes the paper with some remarks.

\section{The Model}

We study the social-network effect on game equilibrium in the context of two-player asymmetric normal-form games. Without great loss of generality, one may interpret our background in the evolutionary sense. That is, there are two heterogeneous groups of populations. In particular, there is a representative row-player with $m$ strategies for the first population, while there is a representative column-player with $n$ strategies available for the second group of population. Naturally, payoffs are determined by two matrices, $A$, which is $m \times n$, for the first population, and $B$, which is $n \times m$, for the second population.

Furthermore, suppose in period $t$ there are $M_{i}$ players who choose strategy $i$ for $\forall i=1, \ldots, m$, and also $N_{j}$ players who choose strategy $j$ for $\forall j=1, \ldots, n$. Thus, we let $x_{i}:=M_{i} / \Sigma M_{k}$ and $y_{j}:=N_{j} / \Sigma N_{l}$ denote the frequencies of strategies $i$ and $j$, respectively, for $\forall i=1, \ldots, m$ and $\forall j=1, \ldots, n$. Thus, applying the random matching rule in a well- mixed population, the average payoffs of strategy $i$ and strategy $j$ are given by $(A y)_{i}$ and $(B x)_{j}$, respectively, for $y:=\left(y_{1}, \ldots, y_{n}\right)^{\prime}$ and $x:=\left(x_{1}, \ldots, x_{m}\right)^{\prime}$ with " ' " denoting transpose. Clearly, we can put,

$$
\begin{aligned}
& \Delta^{\text {row }}:=\left\{x \in \mathbb{R}^{m} \mid \sum_{i=1}^{m} x_{i}=1 \text { for } x_{i} \in[0,1], \forall i=1, \ldots, m\right\} \\
& \Delta^{\text {column }}:=\left\{y \in \mathbb{R}^{n} \text { üiü }_{j=1}^{n} y_{j}=\quad y_{j} \in[0,1], \forall j=1, \ldots, n\right\}
\end{aligned}
$$


It is especially worth emphasizing that $x$ can be regarded as the vector of rowstrategy distribution among the first population in the evolutionary sense on the one hand, it, on the other hand, can also be interpreted as the frequencies of the actions of the representative row-player. Similarly, we can consider the vector $y$ in the same way. Thus, as in Hofbauer and Hopkins (2005), we formally give,

Definition 1 (Belief-learning dynamics in well-mixed populations)

The representative row-player's belief about the actions of the representative columnplayer is characterized by the following learning dynamics,

$$
\dot{y} \in B R(x)-y
$$

where $B R(x)$ is the set of all best responses of column-player to $x \in \Delta^{\text {row }}$. By symmetry, the representative column-player's belief about the actions of the representative row-player is determined by the learning dynamics as follows,

$$
\dot{x} \in B R(y)-x
$$

where $B R(y)$ is the set of all best-response actions of row-player to $y \in \Delta^{\text {column }}$.

Remark 2.1. As is pointed out by Hofbauer and Hopkins (2005), $B R(x)$ and $B R(y)$ are typically not functions but correspondences. Nevertheless, one may also consider some specific best-response functions, for example, the exponential or logit choice rule (see, Hofbauer and Sandholm, 2002),

$$
B R_{j}(x):=\frac{\exp \left[\gamma^{-1}(B x)_{j}\right]}{\sum_{l=1}^{n} \exp \left[\gamma^{-1}(B x)_{l}\right]}, j=1, \ldots, n .
$$

And similarly,

$$
B R_{i}(y):=\frac{\exp \left[\xi^{-1}(A y)_{i}\right]}{\sum_{k=1}^{m} \exp \left[\xi^{-1}(A y)_{k}\right]}, i=1, \ldots, m
$$

where $\gamma, \xi \in(0, \infty)$ denote the noise levels, respectively. And when the noise level approaches zero, logit choice approaches unperturbed maximization; when the noise level approaches infinity, it approaches uniform randomization. Therefore, in well-mixed populations and for the present continuous-time repeated game, one can define,

Problem 1. The optimization problem facing the representative row-player is given by, 


$$
\max _{x \in \Delta^{\prime o w}} \int_{0}^{\infty} e^{-\rho t}(x \cdot A y) d t
$$

subject to,

$$
\dot{y} \in B R(x)-y, y \in \Delta^{\text {column }} .
$$

Problem 2. The optimization problem facing the representative column-player reads as follows,

$$
\max _{x \in \Delta^{\text {column }}} \int_{0}^{\infty} e^{-\rho t}(y \cdot B x) d t
$$

subject to,

$$
\dot{x} \in B R(y)-x, x \in \Delta^{\text {row }}
$$

As one can see, such kind of optimization problem may be involved in optimization subject to non-linear constraints, which hence implies that we generally cannot adopt the neo-classical optimization methodology and dual approach proposed in Ivanov and Dobreva (2010) for the studying of labor supply issues.

Definition 2 (Game equilibrium in a well-mixed population)

If Problem 1 and Problem 2 are solvable, then we denote the corresponding solutions by $x_{y}^{*}$ and $y_{x}^{*}$, respectively. And hence, $\left(x_{y}^{*}, y_{x}^{*}\right)$ is called the game equilibrium in a wellmixed population.

Remark 2.2. Generally speaking, $x_{y}^{*}$ can be regarded as a $C^{r}(r \geq 1)$ map with respect to $y$ and $y_{x}^{*}$ can be regarded as a $C^{r}(r \geq 1)$ map with respect to $x$.

In the above constructions, we just consider the ideal case of well-mixed populations. However, in reality, individuals live in a structured society. That is, there must exist social-network effect which indeed affects the payoffs of the players. In particular, in the current study we incorporate social-network effect by two vectors, $\beta:=\left(\beta_{i}\right)_{i=1, \ldots, m}$ and $\eta:=\left(\eta_{j}\right)_{j=1, \ldots, n}$ with,

$$
\begin{gathered}
\Gamma^{\text {row }}:=\left\{\beta \in \mathbb{R}^{m} \mid \sum_{i=1}^{m} \beta_{i}=0 \text { for } \beta_{i} \in[-1,1], \forall i=1, \ldots, m\right\} \\
\Gamma^{\text {column }}:=\left\{\eta \in \mathbb{R}^{n} \mid \sum_{j=1}^{n} \eta_{j}=0 \text { for } \eta_{j} \in[-1,1], \forall j=1, \ldots, n\right\}
\end{gathered}
$$

denoting the corresponding domains, respectively. As you can see, we characterize the social-network effect from the perspective that social-network structure affects the 
frequencies individuals meet each other in the underlying game. For example, in social interactions, people usually have much higher frequencies to interact with families and neighbors than the remaining people in a given community. What is more, individuals have higher frequencies to interact with people who live in the same community than the people live in any other remaining communities. So, any social-network structure produces the corresponding social-network effect either through the spatial factors such as communities and neighborhoods or based on social collections such as roommate relationship and friendship among the individuals. And these social phenomena sufficiently capture the intuition and essence of our definition of the social-network effect in the model.

Now, with the exogenous social-network effect defined above, the average payoffs of strategies $i$ and $j$ are respectively given by $(A(y+\eta))_{i}$ and $(B(x+\beta))_{j}$, for $i=1, \ldots, m$ and $j=1, \ldots, n$. We, by modifying Definition 1 , give,

Definition 3 (Belief-learning dynamics in social networks)

The representative row-player's belief about the actions of the representative columnplayer is characterized by the following learning dynamics,

$$
\dot{y} \in B R(x+\beta)-y
$$

where $B R(x+\beta)$ is the set of all best responses of column-player to $x+\beta \in \Delta^{\text {row }}$ with $\beta \in \Gamma^{\text {row }}$. Correspondingly, the representative column-player's belief about the actions of the representative row-player is determined by the dynamics as follows,

$$
\dot{x} \in B R(y+\eta)-x
$$

where $B R(y+\eta)$ denotes the set of all best-response actions of the row-player to $y+\eta \in \Delta^{\text {column }}$ with $\eta \in \Gamma^{\text {column }}$.

Accordingly, provided the above preparations, we can give,

Problem 3. The optimization problem, modified by the social-network effect, facing the representative row-player is given by,

$$
\max _{x \in \Delta^{\prime o w}} \int_{0}^{\infty} e^{-\rho t}[(x+\beta) \cdot A(y+\eta)] d t
$$

subject to,

$$
\left\{\begin{array}{c}
\dot{y} \in B R(x+\beta)-y, \quad y \in \Delta^{\text {column }} \\
y+\eta \in \Delta^{\text {column }}, \eta \in \Gamma^{\text {column }} \\
x+\beta \in \Delta^{\text {row }}, \beta \in \Gamma^{\text {row }}
\end{array}\right.
$$

Problem 4. The optimization problem facing the representative column-player in a social network reads as follows, 


$$
\max _{y \in \in^{\text {colum }}} \int_{0}^{\infty} e^{-\rho t}[(y+\eta) \cdot \mathrm{B}(x+\beta)] d t
$$

subject to,

$$
\left\{\begin{array}{c}
\dot{x} \in B R(y+\eta)-x, x \in \Delta^{\text {row }} \\
x+\beta \in \Delta^{\text {row }}, \beta \in \Gamma^{\text {row }} \\
y+\eta \in \Delta^{\text {column }}, \eta \in \Gamma^{\text {column }}
\end{array}\right.
$$

Definition 4 (Game equilibrium in a social network)

Provided Problem 3 and Problem 4 are solvable, we denote the corresponding solutions by $\hat{x}_{y}(\beta, \eta)$ and $\hat{y}_{x}(\eta, \beta)$, respectively. Thus, the pair $\left(\hat{x}_{y}(\beta, \eta), \hat{y}_{x}(\eta, \beta)\right)$ is named as the game equilibrium in a social network.

Remark 2.3. Without loss of generality, $\hat{x}$ can be regarded as a $C^{r}(r \geq 1)$ map with respect to $y$ and also $\hat{y}$ can be seen as a $C^{r}(r \geq 1)$ map with respect to $x$ based upon our constructions. Noting that the key issue of the current study is not the existence of game equilibrium defined above but the social-network effect imposed on the game equilibrium, we suppose throughout that the game equilibria exist with the corresponding $C^{r}(r \geq 1)$ properties fulfilled. And we leave the investigation of the open question about the existence of game equilibrium to future work.

Additionally, in order to verify that Definition 4 is actually well-defined we will introduce the following numerical example to reveal the corresponding desirability.

Example 1. Suppose that the payoff matrices have the following numerical characteristic,

$$
A=\left(\begin{array}{ll}
1 & 0 \\
0 & 2
\end{array}\right), B=\left(\begin{array}{ll}
3 & 0 \\
0 & 1
\end{array}\right)
$$

which shows that we are considering an asymmetric coordination game, i.e., a normal-form game that is widely used and applied in game theory and economic theory. Thus, for the representative row-player in Problem 3, we have,

$$
\begin{aligned}
& (x+\beta) \cdot A(y+\eta)=\left(x_{1}+\beta_{1}, 1-x_{1}+\beta_{2}\right)\left(\begin{array}{ll}
1 & 0 \\
0 & 2
\end{array}\right)\left(\begin{array}{l}
y_{1}+\eta_{1} \\
y_{2}+\eta_{2}
\end{array}\right)= \\
& =\left(x_{1}+\beta_{1}\right)\left(y_{1}+\eta_{1}\right)+2\left(1-x_{1}+\beta_{2}\right)\left(y_{2}+\eta_{2}\right)
\end{aligned}
$$

About the underlying belief-learning dynamics, we specifically choose the broadly employed logit choice rule that is introduced in Remark 2.1, i.e., 


$$
B R_{1}(x+\beta)=\frac{\exp \left[\gamma^{-1}(B(x+\beta))_{1}\right]}{\sum_{l=1}^{2} \exp \left[\gamma^{-1}(B(x+\beta))_{l}\right]}=\frac{1}{1+\exp \left[\gamma^{-1}\left(1+\beta_{2}-3 \beta_{1}-4 x_{1}\right)\right]}
$$

for $\gamma \in(0, \infty)$. In particular, we adopt the following learning process,

$$
d y_{1}=\left(B R_{1}(x+\beta)-y_{1}\right) d t+\sigma_{1} y_{1} d W
$$

where $W$ denotes a standard Brownian motion, i.e., we consider the case of stochastic learning dynamics driven by stochastic replicator dynamics. Hence, the optimization problem facing the representative row-player can be written as follows,

$$
\max _{0 \leq x_{1} \leq 1} \int_{0}^{\infty} e^{-\rho t}\left[\left(x_{1}+\beta_{1}\right)\left(y_{1}+\eta_{1}\right)+2\left(1-x_{1}+\beta_{2}\right)\left(1-y_{1}+\eta_{2}\right)\right] d t
$$

subject to,

$$
d y_{1}=\left(\frac{1}{1+\exp \left[\gamma^{-1}\left(1+\beta_{2}-3 \beta_{1}-4 x_{1}\right)\right]}-y_{1}\right) d t+\sigma_{1} y_{1} d W
$$

The corresponding Bellman equation can be expressed as follows,

$$
\begin{aligned}
& \rho J\left(y_{1}\right)-\frac{1}{2} \sigma_{1}^{2} y_{1}^{2} J^{\prime \prime}\left(y_{1}\right)= \\
& =\max _{0 \leq x_{1} \leq 1}\left\{\left[\left(x_{1}+\beta_{1}\right)\left(y_{1}+\eta_{1}\right)+2\left(1-x_{1}+\beta_{2}\right)\left(1-y_{1}+\eta_{2}\right)\right]+\right. \\
& \left.+J^{\prime}\left(y_{1}\right)\left(\frac{1}{1+\exp \left[\gamma^{-1}\left(1+\beta_{2}-3 \beta_{1}-4 x_{1}\right)\right]}-y_{1}\right)\right\}
\end{aligned}
$$

with $J\left(y_{1}\right)$ representing the value function, which is a $C^{r}(r \geq 1)$ map in this formulation. Therefore, optimal choice of $x_{1}$ is determined by the following first-order condition,

$$
\left(y_{1}+\eta_{1}\right)-2\left(1-y_{1}+\eta_{2}\right)+J^{\prime}\left(y_{1}\right) \frac{4 \gamma^{-1} \exp \left[\gamma^{-1}\left(1+\beta_{2}-3 \beta_{1}-4 x_{1}\right)\right]}{\left(1+\exp \left[\gamma^{-1}\left(1+\beta_{2}-3 \beta_{1}-4 x_{1}\right)\right]\right)^{2}}=0
$$

which is equivalent to the following equation,

$$
\frac{\exp \left[\gamma^{-1}\left(1+\beta_{2}-3 \beta_{1}-4 x_{1}\right)\right]}{\left(1+\exp \left[\gamma^{-1}\left(1+\beta_{2}-3 \beta_{1}-4 x_{1}\right)\right]\right)^{2}}=\frac{\gamma\left[2\left(y_{2}+\eta_{2}\right)-\left(y_{1}+\eta_{1}\right)\right]}{4 J^{\prime}\left(y_{1}\right)}
$$


which implies that $\hat{x}$ is indeed a $C^{r}(r \geq 1)$ map with respect to $y$ and it can be expressed as $\hat{x}_{y}(\beta, \eta)$. Similarly, one can also demonstrate that $\hat{y}_{x}(\eta, \beta)$ is indeed a $C^{r}(r \geq 1)$ map with respect to $x$. And we leave the details to the interested reader. To summarize, game equilibrium in Definition 4 is well-defined and the existence of such kind of game equilibrium endogenously affected by social networks is confirmed in such a numerical example.

\section{Social-Network Effect}

Based on Definition 2 and Definition 4, we give,

$$
f_{y}\left(\beta, \eta ; x_{y}^{*}\right):=\hat{x}_{y}(\beta, \eta)-x_{y}^{*}
$$

which is a $C^{r}(r \geq 1)$ map with respect to its arguments based upon our specifications.

Assumption 1. $0 \leq \operatorname{dim} \Gamma^{\text {row }}+\operatorname{dim} \Gamma^{\text {column }}<\operatorname{dim} \Delta^{\text {row }}$.

From Assumption 1 and also the following assumptions, one can easily find that the present model mainly focuses on mixed-strategy equilibrium emphasized by the seminal papers of Harsanyi (1973) and Fudenberg and Kreps (1993), and among others.

Transversality will be sufficiently used in the following proof. About the definition of transversality, one can refer to Marsden et al. (2001, pp. 179), and one can refer to Hirsch (1976, pp. 74) about the Transversality Theorem and refer to Hirsch (1976, pp. 7980) about the Parametric Transversality Theorem. Moreover, about Preimage Theorem, one can refer to Guillemin and Pollack (1974, pp. 21). And we bring the idea employed by Citanna and Siconolfi (2010) to our major proof.

\section{Proposition 1}

Let $y \in \Delta^{\text {column }}$ be given. Thus, there is an open and dense subset $\Delta^{\text {row* }}$ of $\Delta^{\text {row }}$ such that the system $f_{y}\left(\beta, \eta ; x_{y}^{*}\right)=0$ does not have a solution in the space $\Gamma^{\text {row }} \times \Gamma^{\text {column }}$ for all $x_{y}^{*} \in \Delta^{\text {row* }}$ when Assumption 1 holds.

\section{Proof:}

For $x_{y}^{*} \in \Delta^{\text {row }}, f_{y, x_{y}^{*}}: \Gamma^{\text {row }} \times \Gamma^{\text {column }} \rightarrow \Delta^{\text {row }}$, where we, by Assumption 1, have $\operatorname{dim} \Gamma^{\text {row }}+\operatorname{dim} \Gamma^{\text {column }}<\operatorname{dim} \Delta^{\text {row }}$, and thus there are fewer unknowns than equations. The Jacobian of the map $f_{y}(\cdot)$ with respect to $x_{y}^{*}=\left(x_{y i}^{*}\right)_{i=1, \ldots, m}$ is equal to the negative identity matrix. Hence, $\operatorname{rank} J f_{y}=\operatorname{dim} \Delta^{\text {row }}$, which implies that $f_{y}(\cdot)$ is a $C^{r}(r \geq 1)$ submersion. Now, applying the definition of Transversality produces $f_{y} \pitchfork S$ for $\forall S \subset \Delta^{\text {row }}$. Therefore, Parametric Transversality Theorem implies that for $x_{y}^{*} \in \ddot{\mathrm{A}}^{\text {row* }}$, a dense subset of $\Delta^{\text {row }}$, $f_{y, x_{y}^{*}} \pitchfork S$ for $\forall S \subset \Delta^{\text {row }}$. By Assumption 1, we get $\operatorname{dim} \Gamma^{\text {row }}+\operatorname{dim} \Gamma^{\text {column }}+\operatorname{dim}\{0\}<\operatorname{dim} \Delta^{\text {row }}$, thus we get $f_{y, x_{y}^{*}}\left(\Gamma^{\text {row }} \times \Gamma^{\text {column }}\right) \cap\{0\}=\varnothing$ for $\forall y \in \Delta^{\text {column }}$ and $x_{y}^{*} \in \Delta^{\text {row } w^{*}}$ by using the 
definition of Transversality again. Accordingly, $f_{y}\left(\beta, \eta ; x_{y}^{*}\right):=\hat{x}_{y}(\beta, \eta)-x_{y}^{*}=0$ does not have a solution in the compact product-space $\Gamma^{\text {row }} \times \Gamma^{\text {column }}$ for $\forall y \in \Delta^{\text {column }}$ and $x_{y}^{*} \in \Delta^{\text {row* }}$. Moreover, we proceed to show that $\Delta^{r o w^{*}}$ is also open. Notice that $\{0\}$ is a closed subset provided the usual topology on the simplex $\Delta^{\text {row }}$, and also the map $f_{y}(\cdot)$ is $C^{r}(r \geq 1)$, an application of the Parametric Transversality Theorem produces the required assertion.

Similarly, given,

Assumption 2. $0 \leq \operatorname{dim} \Gamma^{\text {row }}+\operatorname{dim} \Gamma^{\text {column }}<\operatorname{dim} \Delta^{\text {column }}$.

We derive the following proposition,

\section{Proposition 2}

Let $x \in \Delta^{\text {row }}$ be given. Thus, there is an open and dense subset $\Delta^{\text {column }}{ }^{*}$ of $\Delta^{\text {column }}$ such that the system $g_{x}\left(\eta, \beta ; y_{x}^{*}\right):=\hat{y}_{x}(\eta, \beta)-y_{x}^{*}=0$ does not have a solution in the space $\Gamma^{\text {column }} \times \Gamma^{\text {row }}$ for all $y_{x}^{*} \in \Delta^{\text {column }}$ when Assumption 2 holds.

\section{Proof:}

It is easily seen that the proof is quite similar to that of Proposition 1, thus we omit it.

Definition 5 (Independence of game equilibrium)

We mean independence of game equilibrium in social networks in the following sense: the corresponding game equilibrium essentially changes when a non-trivial social-network structure is imposed on the underlying well-mixed population. That is, independence of game equilibrium in social networks implies that we can hardly approximate the game equilibrium in social networks through that relatively easily derived in well-mixed populations and this definition of independence has nothing to do with that of probabilistic independence.

To summarize, we can establish,

Theorem 1 (Independence)

Provided the above constructions, social-network effect indeed generates nontrivial differences among the resulting mixed-strategy game equilibria when the corresponding dimensional constraints in Assumption 1 and Assumption 2 are fulfilled.

Remark 3.1. Theorem 1 implies that, for some important and also interesting cases, one can hardly approximate the game equilibrium in social networks via that in a well-mixed population by using the random-matching rule. Although the methodology of random matching (e.g., Gilboa and Matsui, 1992; Aliprantis et al., 2007, and among others) indeed plays a crucial role in equilibrium selection of (evolutionary) game theory, 
it would probably provide us with wrong or biased predictions about the equilibrium behaviors of individuals in many important and also interesting social networks discussed in Theorem 1. For example, we may conjecture that the equilibrium derived by randommatching mechanism in well-mixed populations cannot approach the equilibrium in social networks even when the corresponding social network approaches the state of well-mixed population provided the social-network effect defined above vanishes. In other words, the game equilibrium in social networks should be of independent interest. Moreover, this result holds with certain stability and also in generic sense thanks to the well-known Transversality Theorem.

Now, we are encouraged to consider the following $C^{r}(r \geq 1)$ map,

$$
\varphi\left(\left(\beta^{1}, \eta^{1}\right),\left(\beta^{2}, \eta^{2}\right) ; y\right):=\hat{x}^{1}\left(\beta^{1}, \eta^{1} ; y\right)-\hat{x}^{2}\left(\beta^{2}, \eta^{2} ; y\right)
$$

for the game equilibria $\hat{x}^{1}(\cdot), \hat{x}^{2}(\cdot) \in \Delta^{\text {row }}$ from Definition 4 and any action $y \in \Delta^{\text {column }}$. And we introduce the following assumption,

Assumption 3. $2\left(\operatorname{dim} \Gamma^{\text {row }}+\operatorname{dim} \Gamma^{\text {column }}\right)<\operatorname{dim} \Delta^{\text {row }}$.

\section{Proposition 3}

Based upon Assumption 3 and the above specifications, there is an open and dense subset $\hat{\Delta}^{\text {column }}$ of $\Delta^{\text {column }}$ such that the system $\varphi\left(\left(\beta^{1}, \eta^{1}\right),\left(\beta^{2}, \eta^{2}\right) ; y\right)=0$ does not have a solution in the set,

$$
\text { Ù : }=\left\{\left(\left(\beta^{1}, \eta^{1}\right),\left(\beta^{2}, \eta^{2}\right)\right) \in \Gamma^{\text {row }} \times \Gamma^{\text {column }} \times \Gamma^{\text {row }} \times \Gamma^{\text {column }}\left\|\mid\left(\beta^{1}, \eta^{1}\right)-\left(\beta^{2}, \eta^{2}\right)\right\| \neq 0\right\}
$$

for $\forall y \in \hat{\Delta}^{\text {column }}$ when the derivative of the map $\varphi$ with respect to $y$ is surjective.

\section{Proof:}

We first put,

$$
\Omega:=\left\{\left(\left(\beta^{1}, \eta^{1}\right),\left(\beta^{2}, \eta^{2}\right)\right) \in \Gamma^{\text {row }} \times \Gamma^{\text {column }} \times \Gamma^{\text {row }} \times \Gamma^{\text {column }}\left\|\left(\beta^{1}, \eta^{1}\right)-\left(\beta^{2}, \eta^{2}\right)\right\| \neq 0\right\}
$$

And for any integer $k>0$, let,

$$
\Omega_{k}:=\left\{\left(\left(\beta^{1}, \eta^{1}\right),\left(\beta^{2}, \eta^{2}\right)\right) \in \Gamma^{\text {row }} \times \Gamma^{\text {column }} \times \Gamma^{\text {row }} \times \Gamma^{\text {column }}\left\|\left(\beta^{1}, \eta^{1}\right)-\left(\beta^{2}, \eta^{2}\right)\right\| \geq \frac{1}{k}\right\}
$$

Obviously, $\Omega_{k} \subset \Omega$, and both $\Omega_{k}$ and $\Omega$ are sets that are (locally) independent of $y \in \Delta^{\text {column }}$. Let $\Delta^{\text {column }}(k)$ denote the subset of $\Delta^{\text {column }}$ where the system $\varphi\left(\left(\beta^{1}, \eta^{1}\right),\left(\beta^{2}, \eta^{2}\right) ; y\right)=0$ does not have a solution in $\Omega_{k}$. If $\Delta^{\text {column }}(k)$ is open and dense in $\Delta^{\text {column }}$, then we obtain, 


$$
\hat{\Delta}^{\text {column }}:=\bigcap_{k>0} \Delta^{\text {column }}(k)
$$

which is the intersection of a countable family of open and dense sets; therefore, it is a residual and therefore dense subset of $\Delta^{\text {column }}$ by applying Baire Category Theorem. And also, the system $\varphi\left(\left(\beta^{1}, \eta^{1}\right),\left(\beta^{2}, \eta^{2}\right) ; y\right)=0$ does not have a solution in $\mathrm{cl} \Omega$, i.e., the closure of the set $\Omega$, for $\forall y \in \hat{\Delta}^{\text {column }}$. Suppose not, then there is $y \in \hat{\Delta}^{\text {column }}$ and $\left(\left(\beta^{1}, \eta^{1}\right),\left(\beta^{2}, \eta^{2}\right)\right) \in \operatorname{cl} \Omega$ such that $\varphi\left(\left(\beta^{1}, \eta^{1}\right),\left(\beta^{2}, \eta^{2}\right) ; y\right)=0$. By the definition of the space $\Omega$, there must exist $\tilde{k}>0$ such that $\left(\left(\beta^{1}, \eta^{1}\right),\left(\beta^{2}, \eta^{2}\right)\right) \in \Omega_{\tilde{k}}$. However, the latter implies that $y \notin \Delta^{\text {column }}(\tilde{k})$, a contradiction.

The compactness of $\Omega_{k}$ implies that $\left\|\varphi\left(\left(\beta^{1}, \eta^{1}\right),\left(\beta^{2}, \eta^{2}\right) ; y\right)\right\| \geq \zeta$ for some $\zeta>0$ and all $\left(\left(\beta^{1}, \eta^{1}\right),\left(\beta^{2}, \eta^{2}\right)\right) \in \Omega_{k}$. However, the map $\varphi(\cdot)$ is continuous in all its arguments and hence $\left\|\varphi\left(\left(\beta^{1}, \eta^{1}\right),\left(\beta^{2}, \eta^{2}\right) ; y\right)\right\|>0$ for all $\left(\left(\beta^{1}, \eta^{1}\right),\left(\beta^{2}, \eta^{2}\right)\right) \in \Omega_{k}$ and $\breve{y}$ in an open neighborhood of $y$. Therefore, it is confirmed that the set $\Delta^{\text {column }}(k)$ is open.

Now, we are in the position to show that $\Delta^{\text {column }}(k)$ is also dense. It follows from Assumption 3 that $\operatorname{dim} \Omega_{k}<\operatorname{dim} \Delta^{\text {row }}$, and thus there are more equations than unknowns in the system $\varphi\left(\left(\beta^{1}, \eta^{1}\right),\left(\beta^{2}, \eta^{2}\right) ; y\right)=0$ for any given $y \in \Delta^{\text {column }}$. Consequently, by the Preimage Theorem and Parametric Transversality Theorem, there is a dense subset $\Delta^{\text {column }}(k)$ of $\Delta^{\text {column }}$ where $\varphi\left(\left(\beta^{1}, \eta^{1}\right),\left(\beta^{2}, \eta^{2}\right) ; y\right)=0$ has no solution in $\Omega_{k}$ due to the assumption that the derivative of the map $\varphi$ with respect to $y$ is surjective. So, the proof is completed.

Remark 3.2. Here, the metric or norm $\|\cdot\|$ is the canonical metric in $\mathbb{R}^{k}$.

Assumption 4. $2\left(\operatorname{dim} \Gamma^{\text {row }}+\operatorname{dim} \Gamma^{\text {column }}\right)<\operatorname{dim} \Delta^{\text {column }}$.

\section{Proposition 4}

Based uponAssumption 4 and the above specifications, there is an open and dense subset $\hat{\Delta}^{\text {row }}$ of $\Delta^{\text {row }}$ such that the system $\psi\left(\left(\eta^{1}, \beta^{1}\right),\left(\eta^{2}, \beta^{2}\right) ; x\right) \fallingdotseq \hat{y}^{1}\left(\eta^{1}, \beta^{1} ; x\right)-\hat{y}^{2}\left(\eta^{2}, \beta^{2} ; x\right)=0$ does not have a solution in the set

$$
\Omega \fallingdotseq\left\{\left(\left(\beta^{1}, \eta^{1}\right),\left(\beta^{2}, \eta^{2}\right)\right) \in \Gamma^{\text {row }} \times \Gamma^{\text {column }} \times \Gamma^{r o w} \times \Gamma^{\text {column }}\left\|\left(\beta^{1}, \eta^{1}\right)-\left(\beta^{2}, \eta^{2}\right)\right\| \neq 0\right\}
$$

for $\forall x \in \hat{\Delta}^{\text {row }}$ when the derivative of the map $\psi$ with respect to $x$ is surjective.

\section{Proof:}

One can easily notice that the proof is quite similar to that of Proposition 3, we take it as omitted and leave it to the interested reader. 
Definition 6 (Uniqueness of game equilibrium)

We discuss uniqueness of game equilibrium in a given social network. For a given social network, the corresponding game equilibrium is a map of the underlying socialnetwork effect (or social-network structure). If different social-network effects lead to different game equilibria or equivalently the same game equilibrium implies that there exists the same social-network effect, we get the game equilibrium as an injective map of the social-network effect. As a result, injection means uniqueness of the game equilibrium in a given social network.

Theorem 2 (Uniqueness)

The game equilibrium given in Definition 4 is injective with respect to the socialnetwork effect when either Proposition 3 or Proposition 4 holds. This yields that different social-network effects produce effective differences among the resulting game equilibria when the corresponding dimensional constraints are satisfied. Thus, naturally, there exists a one-to-one correspondence between the social-network structure and the mixed-strategy equilibrium. That is to say, the uniqueness of the mixed-strategy game equilibrium in a given social network is identified.

Remark 3.3. It is especially worth noting that the above result holds with certain stability and also in generic sense owing to the Transversality Theorem. By this theorem, one can conclude that different social networks would be of independent interest if they indeed produce different social-network effects in the sense of our specification. To sum up, social-network mechanism provides a unique prediction of the equilibrium behaviors of the individuals involved in the underlying game.

\section{Corollary 1}

The property Uniqueness in Theorem 2 implies the property Independence established in Theorem 1.

\section{Proof:}

It is easily seen that Assumption 3 implies Assumption 1 and also Assumption 4 implies Assumption 2, which accordingly yields the required result.

Remark 3.4. This observation also demonstrates the inherent consistency of the underlying model specification. Notice that the above assumptions have provided the minimum requirements of the corresponding dimensional constraints of properties Independence and Uniqueness, we have thus shown the biggest sets of social networks in which Independence and Uniqueness hold true, respectively. 


\section{Conclusion}

In current study, topological analyses about the social-network effect on game equilibrium have been thoroughly provided. It would be very interesting to explore the game equilibrium in social networks rather than well-mixed populations (see, Weibull, 1995; Hofbauer and Sigmund, 2003, and among others), and the paper provides a simple and general framework for this issue. Nevertheless, we just study exogenous social-network effect in the sense of Skyrms and Pemantle (2000) in the present limited model. Moreover, the paper mainly focuses on mixed-strategy equilibrium emphasized by the seminal papers of Harsanyi (1973) and Fudenberg and Kreps (1993).

Two major conclusions are established in the model. Firstly, generally speaking, nontrivial social network induces game equilibrium strictly different from that in wellmixed populations. Secondly, it is interesting to find that the game equilibrium exhibits injective property with respect to the social-network effect under consideration. That is, we have proved the uniqueness of mixed-strategy game equilibrium in a given social network. Therefore, we argue that the game equilibrium in social networks would be of independent interest and random-matching rule (see, Ellison, 1994; Okuno-Fujiwara and Postlewaite, 1995; Weibull, 1995; Hofbauer and Sigmund, 2003, and among others) cannot always provide us with a compelling approximation. Finally, it is shown that uniqueness implies independence for a wide range of social networks. And we have even derived the biggest sets of social networks in which independence and uniqueness hold true, respectively, in the underlying game.

What are the economic implications of the main results established in the paper? On the one hand, even though the theory of game equilibrium in well-mixed populations has been thoroughly established in the past several decades and the importance of socialnetwork effect imposed on game equilibria and economic outcomes has been sufficiently emphasized in recent studies, there still is not a general conclusion regarding the internal relation between the both. The paper demonstrates an impossibility theorem by confirming the independence of game equilibrium in social networks. This impossibility theorem argues that we can hardly predict the equilibrium behaviors in social networks when we only have information about the original well-mixed populations. That is to say, since the players choose their best strategies based on the information of the game context, our result implies that social-network structures produce relevant information that is essential in determining equilibrium behaviors. Rather, one may even argue that game equilibria in social networks are of independent interest mainly because social networks themselves produce informational frictions facing the players when compared to the original wellmixed world. On the other hand, uniqueness of game equilibrium in a given social network not only leads us to the corresponding independence of game equilibrium but also makes things much easier when evaluating economic welfare of different social networks. Noting that we can comparatively easily Pareto rank different game equilibrium according to the corresponding payoffs, we can thus Pareto rank different social networks by applying the uniqueness property. As is well known, social networks are usually formed by social 
norms, conventions and lor institutions. Consequently, one can directly Pareto rank different social norms or institutions based upon our general result. In particular, we have to some extent modeled the underlying idea of Coase (1988) that we need a baseline framework to comparatively and sufficiently evaluate the economic efficiency of different institutional arrangements in order to make a wise choice during the corresponding institutional changes in reality.

As a final point, I'd like to cite some examples in existing articles to make our general arguments much more intuitive. First, the simple model constructed in Dai and Cheng (2011) can be regarded as a special application of the Independence property demonstrated in this paper. As is widely known, (Defect, Defect) is the unique Nash equilibrium and evolutionary stable equilibrium (ESE) of Prisoner's Dilemma in a well-mixed population (see, Weibull, 1995; Hofbauer and Sigmund, 2003, and among others). However, Dai and Cheng (2011) prove that there is a non-random matching mechanism, which naturally corresponds to a special type of social-network structure (or effect), such that (Cooperate, Cooperate) is the unique induced game equilibrium. That is to say, social-network effect does make sense and game equilibria in some social networks are of independent interest relative to those in well-mixed populations. Second, as is emphasized above, Uniqueness not only implies Independence but also yields interesting economic-welfare implications. Dai (2012) indeed reveals a general existence of the Pareto-optimal social-network structure in any given evolutionary normal-form game. Moreover, in a much simpler example, Dai and Cheng (2011) prove that there exists an optimal and stable level of social segmentation, which also results in a special type of social network, so that the welfare of the community is maximized under the background of Prisoner's Dilemma. Notice that Dai and Cheng (2011), and Dai (2012) only confirm the existence of Pareto-optimal social networks in evolutionary normal-form games, the present paper further demonstrates the underlying Uniqueness property, thereby making the Pareto ranking of different social networks much easier in large and general normal-form games. As a consequence, what are the corresponding lessons we have learned from this paper? On the one hand, like rational principle, evolutionary selection and learning mechanism, we can similarly use social-network effect as an effective equilibrium-selection mechanism especially when there are multiple equilibria in many social games or spatial games. On the other hand, the general result established in the paper also shows that we can design a unique socialnetwork structure through formal social institutions or informal social norms to induce the Pareto-optimal game equilibrium of structured populations in real-world economies.

\section{Acknowledgment}

I am very grateful for helpful comments and suggestions, which I believe have greatly improved the quality of the paper, from the anonymous referee. Any remaining errors are, of course, my own responsibility. 


\section{References}

Acemoglu, D., Dahleh, M., Lobel, I. and Ozdaglar, A., 2011, 'Bayesian Nearning in Social Networks', Review of Economic Studies, 78, pp. 1201-1236.

Acemoglu, D., Carvalho, V. M., Ozdaglar, A. and Tahbaz-Salehi, A., 2012, 'The Network Origins of Aggregate Fluctuations', Econometrica, 80, pp. 1977-2016.

Aliprantis, C. D., Camera, G. and Puzzello, D., 2007, 'A Random Matching Theory', Games and Economic Behavior, 59, pp. 1-16.

Allen, B., Traulsen, A., Tarnita, C. E. and Nowak, M. A., 2012, 'How Mutation Affects Evolutionary Games on Graphs', Journal of Theoretical Biology, 299, pp. 97-105.

Anderson, A. and Smith, L., 2010, 'Dynamic Matching and Evolving Reputations', Review of Economic Studies, 77, pp. 3-29.

Bala, V. and Goyal, S., 2000, 'A Noncooperative Model of Network Formation', Econometrica, 68, pp. 1181-1229.

Bandiera, O. and Rasul, I., 2006, 'Social Networks and Technology Adoption in Northern Mozambique', Economic Journal, 116, pp. 869-902.

Bowles, S. and Gintis, H., 1998, 'The Moral Economy of Communities: Structured Populations and the Evolution of Pro-Social Norms', Evolution and Human Behavior, 19 , pp. 3-25.

Citanna, A. and Siconolfi, P., 2010, 'Recursive Equilibrium in Stochastic OverlappingGenerations Economies', Econometrica, 78, pp. 309-347.

Coase, R. H., 1988, 'The Firm, the Market and the Law', Chicago: University of Chicago Press.

Dai, D., and Cheng, Y., 2011, 'Asymmetry and Endogenous Cooperation in Prisoners' Dilemma Game', SSRN Working Paper.

Dai, D., 2012, 'On the Existence of Pareto Optimal Endogenous Matching', Economic Research Guardian, 2, pp. 201-224.

Ellison, G., 1993, 'Learning, Local Interaction, and Coordination', Econometrica, 61, pp. 1047-1071.

Ellison, G., 1994, 'Cooperation in the Prisoner's Dilemma with Anonymous Random Matching', Review of Economic Studies, 61, pp. 567-588.

Fu, F., Nowak, M. A. and Hauert, C., 2010, 'Invasion and Expansion of Cooperators in Lattice Populations: Prisoner's Dilemma vs. Snowdrift Games', Journal of Theoretical Biology, 266, pp. 358-366.

Fudenberg, D. and Kreps, D., 1993, 'Learning Mixed Equilibria', Games and Economic Behavior, 5, pp. 320-367.

Fudenberg, D. and Levine, D. K., 1993, 'Steady State Learning and Nash Equilibrium', Econometrica, 61, pp. 547-573.

Galeotti, A., Goyal, S. and Kamphorst, J., 2006, 'Network Formation with Heterogeneous Players', Games and Economic Behavior, 54, pp. 353-372.

Ghosh, P. and Ray, D., 1996, 'Cooperation in Community Interaction without Information Flows', Review of Economic Studies, 63, pp. 491-519. 
Gilboa, I. and Matsui, A., 1992, 'A Model of Random Matching', Journal of Mathematical Economics, 21, pp. 185-197.

Golub, B. and Jackson, M. O., 2010, 'Naïve Learning in Social Networks and the Wisdom of Crowds', American Economic Journal: Microeconomics, 2, pp. 112-149.

Goyal, S., 2007, 'Connections: an Introduction to the Economics of Networks', Princeton University Press, Princeton, New Jersey.

Guillemin, V. and Pollack, A., 1974, 'Differential Topology', Prentice Hall Inc., New Jersey. Haag, M. and Lagunoff, R., 2006, 'Social Norms, Local Interaction, and Neighborhood Planning', International Economic Review, 47, pp. 265-296.

Harsanyi, J., 1973, 'Games with Randomly Disturbed Payoffs: A New Rationale for MixedStrategy Equilibrium Points', International Journal of Game Theory, 2, pp. 1-23.

Hirsch, M. W., 1976, 'Differential Topology', New York: Springer-Verlag.

Hofbauer, J. and Hopkins, E., 2005, 'Learning in Perturbed Asymmetric Games', Games and Economic Behavior, 52, pp. 133-152.

Hofbauer, J. and Sandholm, W. H., 2002, 'On the Global Convergence of Stochastic Fictitious Play', Econometrica, 70, pp. 2265-2294.

Hofbauer, J. and Sigmund, K., 2003, 'Evolutionary Game Dynamics', Bulletin of the American Mathematical Society, 40, pp. 479-519.

Horst, U. and Scheinkman, J. A., 2006, 'Equilibria in Systems of Social Interactions', Journal of Economic Theory, 130, pp. 44-77.

Ivanov, I. and Dobreva, J., 2010, 'Modeling Labor Supply through Duality and the Slutsky Equation', International Journal of Economic Sciences and Applied Research, 3, 2, pp. 111-122.

Jackson, M. O., 2008, 'Social and Economic Networks', Princeton, NJ: Princeton University Press.

Kandori, M., 1992, 'Social Norms and Community Enforcement', Review of Economic Studies, 59, pp. 63-80.

Kahneman, D., Knetsch, J. L. and Thaler, R., 1986, 'Fairness as a Constraint on Profit Seeking: Entitlements in the Market', American Economic Review, 76, pp. 728-741.

Marsden, J. E., Ratiu, T. and Abraham, R., 2001, 'Manifolds, Tensor Analysis, and Applications', Third Edition, New York: Springer-Verlag.

Mas-Colell, A. and Nachbar, J., 1991, 'On the Finiteness of the Number of Critical Equilibria, with an Application to Random Selections', Journal of Mathematical Economics, 20, pp. 397-409.

Maynard Smith, J., 1982, 'Evolution and the Theory of Games', New York: Cambridge University Press.

Nowak, M. A., 2006, 'Five Rules for the Evolution of Cooperation', Science, 314, pp. $1560-1563$.

Ohtsuki, H., Hauert, C., Lieberman, E. and Nowak, M. A., 2006, 'A Simple Rule for the Evolution of Cooperation on Graphs and Social Networks', Nature, 441, pp. 502-505.

Ohtsuki, H., Nowak, M. A. and Pacheco, J. M., 2007, 'Breaking the Symmetry between 
Interaction and Replacement in Evolutionary Dynamics on Graphs', Physical Review Letters, 98, pp. 108106.

Okuno-Fujiwara, M. and Postlewaite, A., 1995, 'Social Norms and Random Matching Games', Games and Economic Behavior, 9, pp. 79-109.

Pacheco, J. M., Traulsen, A., Ohtsuki, H. and Nowak, M. A., 2008, 'Repeated Games and Direct Reciprocity under Active Linking', Journal of Theoretical Biology, 250, pp. 723731.

Pacheco, J. M., Traulsen, A. and Nowak, M. A., 2006, 'Co-Evolution of Strategy and Structure in Complex Networks with Dynamical Linking', Physical Review Letters, 97, pp. 258103.

Schelling, T., 1969, 'Models of Segregation', American Economic Review, 59, pp. 488-493. Schelling, T., 1971, 'Dynamic Models of Segregation', Journal of Mathematical Sociology, 1, pp. 143-186.

Skyrms, B. and Pemantle, R., 2000, 'A Dynamic Model of Social Network Formation', Proceedings of the National Academy of Sciences of the United States of America, 97, pp. 9340-9346.

Spagnolo, G., 1999, 'Social Relations and Cooperation in Organizations', Journal of Economic Behavior and Organization, 38, pp. 1-25.

Takahashi, S., 2010, 'Community Enforcement when Players Observe Partners' Past Play', Journal of Economic Theory, 145, pp. 42-62.

Tarnita, C. E., Ohtsuki, H., Antal, T., Fu, F. and Nowak, M. A., 2009, 'Strategy Selection in Structured Populations', Journal of Theoretical Biology, 259, pp. 570-581.

Weibull, J., 1995, 'Evolutionary Game Theory', Cambridge, MA: The MIT Press.

Zhang, J., 2004, 'A Dynamic Model of Residential Segregation', Journal of Mathematical Sociology, 28, pp. 147-170. 